\title{
Anabases
}

ANABASES Traditions et réceptions de l'Antiquité

12 | 2010

Varia

\section{Leandro Polverini e la storiografia moderna sul mondo antico}

Gino Bandelli

\section{(2) OpenEdition}

Journals

Edizione digitale

URL: http://journals.openedition.org/anabases/1013

DOI: 10.4000/anabases.1013

ISSN: 2256-9421

Editore

E.R.A.S.M.E.

\section{Edizione cartacea}

Data di pubblicazione: 1 ottobre 2010

Paginazione: 23-43

ISSN: 1774-4296

\section{Notizia bibliografica digitale}

Gino Bandelli, «Leandro Polverini e la storiografia moderna sul mondo antico », Anabases [Online], 12 2010, Messo online il 01 octobre 2013, consultato il 20 octobre 2019. URL : http:// journals.openedition.org/anabases/1013 ; DOI : 10.4000/anabases.1013

(c) Anabases 


\section{Leandro Polverini e la storiografia moderna sul mondo antico*}

GiNO BANDELLI

Durante l'Incontro di studio in onore di Filippo Càssola e Ruggero Fauro Rossi, tenutosi nel novembre del $1998^{1}$, Leandro Polverini dichiarò che il suo apporto a quella giornata doveva intendersi come "un bilancio in itinere» sull'opera di uno dei due Maestri della Scuola triestina di Storia antica ${ }^{2}$. Dodici anni dopo voglio rendere omaggio con il medesimo spirito al Collega e Amico di cui festeggiamo il $75^{\circ}$ compleanno.

Una breve nota, premessa ad un lavoro uscito qualche tempo $\mathrm{fa}^{3}$, ne compendiava in questi termini la produzione scientifica: «I suoi contributi riguardano l'età del passaggio dalla repubblica al principato, vari aspetti del principato e della "crisi del III secolo". Si è occupato, inoltre, di storia locale dell'Italia romana (Firmum Picenum), di storiografia antica (Historia Augusta) e di storiografia moderna sul mondo antico ${ }^{4}$.

* Ringrazio Alessandro Galimberti, Arnaldo Marcone, Pier Giuseppe Michelotto, Massimo Nafissi, Francesco Prontera, Maria Carla Spadoni Cerroni, Alfredo Valvo per gli aiuti che mi hanno prestato in vario modo.

1 G. BANDelli (a cura di), La Scuola triestina di Storia antica, Incontro di studio in onore di Filippo Càssola e Ruggero Fauro Rossi (Trieste, 16 novembre 1998), Quaderni Giuliani di Storia 21 (2000), 2, p. 199-338.

2 Appendice, nr. 43, p. 249.

3 "Germani in Italia prima dei Cimbri?", in B. e P. SCARDigli (a cura di), Germani in Italia, Roma, 1994, p. 1-10.

4 Ibid., p. 1. A quelle concernenti gli àmbiti elencati vanno aggiunte, in particolare, le pubblicazioni sugli spettacoli e sul calendario di Roma. Cf., inoltre, la nota successiva. 
La molteplicità dei temi suddetti (fra cui peraltro non compaiono alcuni di quelli trattati nel Dizionario Epigrafico di Antichità Romane e in altre sedi, come il Grande Dizionario Enciclopedico UTET, l'Enciclopedia Virgiliana, l'Enciclopedia Oraziana) e il numero delle pubblicazioni relative (non meno di 300$)^{5}$ escludono la possibilità di formulare, in questo volume di Anabases, «un bilancio in itinere» di carattere generale. Ho preferito, dunque, limitarmi a un solo aspetto dell'opera di Leandro Polverini, quello concernente la storia della storiografia moderna sul mondo antico: il più nutrito dal punto di vista quantitativo (circa 80 lavori) ${ }^{6}$, uno dei più rilevanti sotto il profilo scientifico e il più vicino, tra i suoi, alle mie competenze.

Nel primo gruppo di titoli del Collega, di qualche anno posteriore alla laurea in lettere classiche (1958), all'inizio dell'attività d'insegnante nella scuola media (1958), al diploma di perfezionamento in Storia romana (1960) e alla borsa Fulbright presso l'American Academy in Rome (1962-1963) ${ }^{7}$, l'interesse per tale filone di ricerca si manifesta precocemente, come dimostrano alcune pagine su Benedetto Croce e su Johann Gustav Droysen, oltre che una rassegna dedicata a lavori teorici e metodologici di Johan Huizinga, Henri-Irénée Marrou, Edward H. Carr e Luigi Bulferetti ${ }^{8}$. È probabile che, a favorire simili aperture, contribuisse anche l'insegnamento di Albino Garzetti, suo Maestro nell'Università Cattolica di Milano', cui egli riconosce, fra i

Non disponiamo ancora di una bibliografia completa dello Studioso: il conteggio che segue, fondato sulla ricognizione che ho potuto fare con l'aiuto suo e di comuni amici, è dunque approssimativo. - Saggi apparsi in varie sedi: 120 circa (56 dei quali su problemi di storia della storiografia moderna sul mondo antico: Appendice, nrr. 1-55). - Voci del D.E.A.R., IV, 3, fasc. 63-68 (1975-1979), passim: 10. - Voci dell'E. V., I-V, 1 (19841990), passim: 29. - Voci del G. D. E. UTET, III-XX (1985-1991), passim: 41 (17 delle quali dedicate a storici dell'Antichità moderni e contemporanei: Appendice, nrr. 56-72). - Voci dell' E. O., I-II (1996-1997), passim: 8. - A tale produzione vanno aggiunte 8 curatele di volumi ( 7 delle quali pertinenti alla storia della storiografia moderna sul mondo antico: Appendice, nrr. 73-79) e 60 e più recensioni.

6 Per un elenco di essi cf. l'Appendice.

7 Desumo queste notizie da un Curriculum vitae di Leandro Polverini. Cf. inoltre: Appendice, nr. 19, p. 298, nota 75 e nr. 43, p. 249 [per la tesi di laurea, successivamente pubblicata: "L'aspetto sociale del passaggio dalla Repubblica al Principato", Aevum 38 (1964), 1-2, p. 241-285; 3-4, p. 439-467; 39 (1965), 1-2, p. 1-24]; A. GarzetTI, "Prefazione", in Contributi dell'Istituto di Filologia classica, Sezione di Storia antica, I, Milano, 1963, p. VIII [per la tesi di perfezionamento, successivamente pubblicata: "Le città dell'Impero nell'epistolario di Plinio", ibid., p. 137-236].

8 Appendice, nrr. 1, 3, 2.

9 Il nome dello Studioso (Bormio, 5 luglio 1914 - Sondalo, 8 luglio 1998) ritorna di frequente nell'opera del discepolo: cf. in particolare, tra i saggi elencati nell'Appendice, quello corrispondente al nr. 42. Inoltre: L. PolverinI, "Cesare e la Britannia", in C. STella, A. Valvo (a cura di), Studi in onore di Albino Garzetti, Brescia, 1996, p. 325-339, in particolare p. 325-326. Un terzo lavoro, intitolato "Gli anni milanesi di Albino Garzetti", è d'imminente pubblicazione. All'interesse generale di Leandro Polverini per la scuola di Plinio Fraccaro dedicherò qualche osservazione più avanti. 
tanti meriti, pure quello di aver destinato uno «spazio relativamente largo» nelle prime lezioni dei suoi corsi alla storiografia moderna sul mondo antico ${ }^{10}$.

Ma la svolta determinante si ebbe, nella nostra prospettiva, con il passaggio di Leandro Polverini all'Istituto Italiano per la Storia Antica, dov'egli, nel frattempo (1963) divenuto professore di ruolo di Latino, Storia e Geografia ${ }^{11}$, ottenne un «comando» (1967-1971) ${ }^{12}$ : in Via Milano conobbe infatti Silvio Accame, direttore dell'Istituto medesimo e depositario dell'archivio di Gaetano De Sanctis.

Del grande patrimonio di scienza e di umanità rappresentato da quest'ultimo Silvio Accame aveva già edito alcune parti: da un lato il I volume degli Scritti minori ${ }^{13}$, dall'altro la versione integrale delle Memorie, una scelta di pagine del Diario e ampi stralci di varie corrispondenze ${ }^{14}$. Un esito immediato dell'inserimento del giovane collaboratore in questa impresa, tanto meritoria quanto ardua, fu la pubblicazione del

10 Appendice, nr. 42, p. 24, nota 31. Cf., in particolare, il capitolo sulla storiografia moderna in A. GARZETTI, Introduzione alla storia romana con un'appendice di esercitazioni epigrafiche, Milano, 1953 (settima edizione: Milano, 1995).

11 Presso l'Istituto Magistrale «Adelaide Cairoli» di Pavia. - Del suo impegno come docente nella scuola secondaria è testimonianza significativa l'articolo "Historia magistra vitae?", Vita e pensiero 49 (1966), p. 734-738.

$12 \mathrm{Al}$ centro di ricerca del quale divenne ospite l'Autore ha dedicato già diversi contributi (Appendice, nrr. 25, 30, 44, p. 159-160); un altro ("La riorganizzazione degli studi storici e l'Istituto Italiano per la Storia Antica") è preannunciato.

13 G. De Sanctis, Scritti minori, a cura di S. Accame, I, Roma, 1966. L'iniziativa proseguì negli anni successivi: G. De Sanctis, Scritti minori, Novamente editi da A. Ferrabino e S. ACCAME, II, 1970, III, 1972, IV, 1976, V, 1983, VI, 1-2, 1972 (sui due tomi dell'ultimo volume: nota 15). Durante la sua realizzazione apparve un altro contributo fondamentale: G. De Sanctis, Storia dei Romani, I, La conquista del primato in Italia, Nuova edizione stabilita sugli inediti a cura di S. ACCAME, Firenze, 1979.

14 Memorie: G. De Sanctis, Ricordi della mia vita, a cura di S. AcCAme, Firenze, 1970, p. 3-4 (Al lettore), p. 5-7 (Premessa), p. 9-13 (La guerra coloniale), p. 15-161 (Note biografiche) (Leandro Polverini segnala che il titolo del manoscritto desanctisiano è Lebenserinnerungen: Appendice, nr. 5, p. 1057, nota 2). - Brani del Diario: ibid., p. 162-235. Un'edizione completa di questo fu pubblicata molto più tardi: G. DE SANCTIS, Il diario segreto (19171933), con introduzione e a cura di S. Accame, Quaderni della Nuova Antologia, LII, Firenze, 1996 (recensito in Appendice, nr. 32). - Lettere pertinenti al rifiuto di prestare il giuramento imposto dal regime fascista ai docenti universitari nel 1931 e agli Accademici dei Lincei nel 1934: De Sanctis, Ricordi, p. 236-255. Tra la fine degli Anni Sessanta e l'inizio degli Anni Settanta varie centinaia di documenti politici ed epistolari desanctisiani, ordinati secondo un criterio tematico, erano apparsi, nel testo completo o in forma parziale, anche in numerosi lavori del suo allievo, elencati ora, con quelli analoghi del periodo successivo (nei quali emerge S. Accame, Gaetano De Sanctis fra cultura e politica. Esperienze di militanti cattolici a Torino 1919-1929, Firenze, 1975), in F. FabBrinI, Silvio Accame studioso del mondo antico, Roma, 2000, p. 531-545 (passim). 
VI volume, in due tomi, degli Scritti minori ${ }^{15}$, seguita da un ampio articolo su Gaetano De Sanctis recensore ${ }^{16}$.

Non molto dopo Leandro Polverini - dal 1969 candidato nell'ultimo concorso per la libera docenza, dal 1970 incaricato di Storia romana alla Facoltà di Lettere e Filosofia di Siena, dal 1971 «comandato» presso la Giunta Centrale per gli Studi Storici ${ }^{17}$ - ebbe la possibilità di accedere all'archivio di Giulio Beloch, allora custodito dalla figlia Margherita ${ }^{18}$. Ne derivò un allargamento di prospettive, che lo indusse ad affrontare anche il problema della fondazione, ad opera dello Studioso tedesco, della Scuola romana di Storia antica ${ }^{19}$, mentre continuava le ricerche sul maggiore dei suoi allievi (premessa, fra l'altro, di nuove operazioni editoriali, come quella relativa al frammento del V volume della Storia dei Romani dedicato alla guerra sociale) ${ }^{20}$.

Il fatto che, da quel momento e sino ad ora, la maggior parte delle pubblicazioni del Collega pertinenti alla storiografia moderna sul mondo antico riguardasse Giulio Beloch (1854-1929) e Gaetano De Sanctis (1870-1957) non ha impedito ch'egli trattasse in modo saltuario anche di autori precedenti, come Stefano Antonio Morcelli (1737-1821) ${ }^{21}$ e Barthold Georg Niebuhr (1776-1831), destinatario di alcune missive di Giacomo Leopardi $(1823,1824,1827)$ e di Bartolomeo Borghesi $(1829)^{22}$, e che

15 Appendice, nr. 73 (dove Appendice, nr. 4: da cui emerge per la prima volta il rigore filologico dell'editore).

16 Appendice, nr. 5 (comprendente anche una scelta di materiali diversi dell'archivio: I, Una recensione al Ciccotti rimasta inedita, p. 1071-1075; II, Dal carteggio Beloch-De Sanctis, p. 1076-1087; III, Recensioni erodotee: commenti di F. Jacoby e M. Pohlenz, p. 1088-1091; IV, La prefazione al Pericle nel carteggio De Sanctis-Principato, p. 1092-1094).

17 La libera docenza in Storia romana gli venne conferita nel 1971; l'incarico proseguì fino al 1977, il «comando» fino al 1980.

18 Appendice, nr. 5, p. 1076, nota 1; nr. 6, p. 153, nota 3; nr. 9, p. 1369, nota 3; nr. 10, p. 1429, nota 1; nr. 17, p. 199-200, nota 1; nr. 20, p. 13, nota 7. - Su Margherita Beloch Piazzolla (1879-1976), da ultimo: Appendice, nr. 29, p. 412, nota 12.

19 Per un elenco dei primi saggi al riguardo cf. Appendice, nrr. 6, 9 (dove Il testo della lezione), 10, 11 (dove la traduzione italiana di buona parte della prefazione russa di Michail Geršenzon), 12.

20 Appendice, nr. 74 (dove Appendice, nr. 8). - Cf., inoltre, i materiali pubblicati nei lavori sulla «rivista storica che non fu mai fondata» (Appendice, nr. 7, dove, p. 429-433, le due stesure - 1912 e 1917 - del Programma concernente la medesima; p. 434-445, i relativi scambi di lettere con - 1912 - Giorgio Pasquali e - 1917 - con Ernesto Bonaiuti, Augusto Rostagni, Nicola Turchi, Federico Halbherr; p. 445-447, il testo - riferibile ad un momento compreso tra il 1944 e il 1947 - degli Elementi per un promemoria sull'utilità d'un insegnamento di metodologia e storia della scienza dell'antichità) e sulla "Storia dei Romani che non fu scritta» (Appendice, nr. 13). - Non meno importante la notizia relativa al Frammento di una dottrina gnoseologica di G. De Sanctis (di epoca non precisata): Appendice, nr. 5, p. 1064, nota 1.

21 Appendice, nr. 21.

22 Appendice, nrr. 18 (con il testo di tre lettere) e 36 (con il testo di una lettera). - Sono di grande interesse anche le annotazioni su Napoleone I lettore di Cesare e su Napoleone 
l'attenzione riservata prioritariamente alla Scuola romana si allargasse ad altre figure legate, per affinità o per contrasto, ai due maggiori esponenti di essa: donde i contributi sulle relazioni fra Giulio Beloch e Theodor Mommsen (1817-1903), Eduard Meyer (1855-1930), Gaetano De Sanctis, Plinio Fraccaro (1882-1959) da un lato, fra Gaetano De Sanctis e Michele Rostovzev (1870-1952), Plinio Fraccaro, Arnaldo Momigliano (1908-1987) dall'altro 23, o i saggi dedicati a Ettore Pais (1854-1929) ed a Guglielmo Ferrero (1871-1942) ${ }^{24}$. Ma lo Studioso ha tracciato anche lucidi profili di altri esponenti dell'antichistica del Novecento, da quello di Pierre Grimal (19121996) ${ }^{25}$ a quelli di Maestri, colleghi e amici, italiani e stranieri, come Albino Garzetti (1914-1998), Massimiliano Pavan (1921-1991), Karl Christ (1923-2008), Ruggero Fauro Rossi (1925-2007) ${ }^{26}$.

L'originalità e il valore di una ricerca dispiegatasi fin dal principio in un contesto nazionale e internazionale ricco di vitalità - i primi saggi di vasto respiro dedicati da Leandro Polverini alla storiografia moderna sul mondo antico risalgono all'inizio degli Anni Settanta, cioè sono più o meno contemporanei all'inaugurazione dei seminari pisani di Arnaldo Momigliano (1972) ${ }^{27}$ - e continuata nel periodo successivo in un quadro non meno favorevole per la nascita di riviste come Quaderni di Storia (1975)

III autore (con il contributo di storici di professione) di un'Histoire de Jules César : Appendice, nr. 46, p. 408-414.

23 Su Beloch in generale: cf. supra, nota 19; inoltre, Appendice, nrr. 20, 24 (dove i programmi di alcuni dei corsi di Geografia storica), 51, 52, 56, 75. Beloch-Mommsen: Appendice, nr. 53 (nei carteggi del Maestro slesiano questa lettera giovanile - 20 novembre 1876 - a colui che sarebbe divenuto il suo più irriducibile avversario è un unicum). Beloch-Meyer: Appendice, nrr. 17, 28, 49. Beloch-De Sanctis: Appendice, nr. 5, p. 10761087. Beloch-Fraccaro: Appendice, nr. 29, p. 411-415. - Su De Sanctis in generale: cf. supra, note 15, 16, 20. De Sanctis-Rostovzev: Appendice, nr. 40 (dove anche un accenno, p. 112, all'esistenza di lettere desanctisiane dell'archivio della Nuova Italia). De SanctisFraccaro: Appendice, nrr. 15 e 29, p. 415-419. De Sanctis-Momigliano: Appendice, nr. 48. - Su Momigliano in generale: Appendice, nrr. 22, 47, 48, 50, 79. Cf., inoltre, le recensioni dedicate, rispettivamente, al Quarto contributo alla storia degli studi classici e del mondo antico, Roma, 1969 [Aevum 43 (1969), 5-6, p. 551], agli Essays in Ancient and Modern Historiography, Oxford, 1977 [Athenaeum n. s., 58 (1980), 3-4, p. 480-483] e al Sesto contributo alla storia degli studi classici e del mondo antico, Roma, 1980 [Athenaeum n. s., 62 (1984), 1-2, p. 340-345].

24 Pais: Appendice, nrr. 44, p. 147-150, 45, 78. - Ferrero: Appendice, nr. 19 (con lettere di Ferrero e Meyer). Cf., inoltre, le osservazioni su Ettore Ciccotti (1863-1939), in Appendice, nr. 5, p. 1071-1075 e su Guido Porzio (1868-1957), in Appendice, nr. 6.

25 Appendice, nr. 33.

26 Garzetti: cf. supra, nota 9. - Pavan: recensione di M. PAVAn, Dall'Adriatico al Danubio, a cura di M. Bonamente e G. Rosada, Padova, 1991, Quaderni di Archeologia del Veneto 8 (1992), p. 243-244; Appendice, nrr. 31 e 41. - Christ: Appendice, nrr. 16, 54, 55. Rossi: Appendice, nr. 43.

27 Dove, nel 1981, Leandro Polverini ebbe l'occasione di presentare in anteprima il carteggio Beloch-Meyer: Appendice, nr. 17, p. 199. - Per un bilancio relativo a quella stagione cf. 
e Storia della Storiografia (1982) e per altre iniziative dello stesso Momigliano, di Karl Christ, di Emilio Gabba - penso ai convegni di Trento (1986) e di Marburg (1988), in cui fu coinvolto anche il nostro Collega ${ }^{28}$ - quell'originalità e quel valore si possono cogliere tanto a livello progettuale, quanto a livello «tecnico».

Nell'àmbito della vicenda suddetta nacque una delle sue realizzazioni più notevoli. Trasferitosi nel 1977 dall'Università di Siena a quella di Perugia (dove subentrò a Massimiliano Pavan nell'insegnamento di Storia romana) e conseguito lo straordinariato nel 1980, l'ordinariato nel 1983, Leandro Polverini trasse vantaggio dalle opportunità offertegli nella nuova sede, "ponte, ideale e concreto, fra la patria toscana e la residenza romana ${ }^{29}{ }_{n}$ : con Gianfranco Maddoli, titolare di Storia greca, diede l'avvio agl' Incontri perugini di storia della storiografia antica e sul mondo antico, inaugurati nel 1986 da un bilancio sull'opera di Giulio Beloch e proseguiti, ben oltre la sua chiamata nel 1990 all'Università di Roma, fino al 2001, con alternanza di temi classici e ottonovecenteschi ${ }^{30}$.

G. Cambiano, "Momigliano e i seminari pisani di storia della storiografia", Storia della Storiografia 8 (1989), 16, p. 75-83.

28 K. Christ, A. Momigliano (a cura di / hrsg. von), L'antichità nell'Ottocento in Italia e Germania / Die Antike im 19. Jahrhundert in Italien und Deutschland, Atti della settimana di studio / Akten der Studienwoche (Trento, 1-5 settembre / Trento, 1.-5. September 1986), Bologna / Berlin, 1988 (dove Appendice, nr. 17). - K. CHrist, E. GABBA (hrsg. von), Römische Geschichte und Zeitgeschichte in der deutschen und italienischen Altertumswissenschaft während des 19. und 20. Jahrhunderts, I, Caesar und Augustus, [Atti del Convegno (Marburg, 4-9 settembre 1988)], Como, 1989 (dove Appendice, nr. 19). Al Convegno di Marburg seguì quello di Pavia: E. GABBA, K. CHRIST (a cura di), Römische Geschichte und Zeitgeschichte in der deutschen und italienischen Altertumswissenschaft während des 19. und 20. Jahrhunderts, II, L'impero romano fra storia generale e storia locale, [Atti del Convegno (Pavia, 3-8 settembre 1989)], Como, 1991.

29 Appendice, nr. 37, p. 63.

30 I: L. Polverini (a cura di), Aspetti della storiografia di Giulio Beloch, 1986 (Appendice, nr. 75); II: G. Maddoli (a cura di), Strabone e l'Italia antica, Acquasparta, 25-27 maggio 1987, Napoli, 1988; III: L. Polverini (a cura di), Lo studio storico del mondo antico nella cultura italiana dell'Ottocento, 1988 (Appendice, nr. 76); IV: F. PronTERA (a cura di), Geografia storica della Grecia antica. Tradizioni e problemi, Acquasparta, 29 maggio - $1^{\circ}$ giugno 1989, Roma-Bari, 1991; V: L. Polverini (a cura di), Erudizione e antiquaria a Perugia nell'Ottocento, 1990 (Appendice, nr. 77); VI: G. Maddoli (a cura di), L'Athenaion politeia di Aristotele 1891-1991. Per un bilancio di cento anni di studi, Acquasparta, 27-29 maggio 1991, Napoli, 1994; VII: L. Polverini (a cura di), Aspetti della storiografia di Ettore Pais, 1992 (Appendice, nr. 78); VIII: M. Gigante, G. MAddoli (a cura di), L’Athenaion politeia dello Pseudo-Senofonte, Acquasparta, 28-30 maggio 1993, Napoli, 1997; IX: A. MArCone (a cura di), Rostovtzeff e l'Italia, Gubbio, 25-27 maggio 1995, Napoli, 1999; X: A. M. Biraschi, G. Salmeri (a cura di), Strabone e l'Asia Minore, Perugia, 25-28 maggio 1997, Napoli, 2000; XI: L. Polverini (a cura di), Arnaldo Momigliano nella storiografia del Novecento, 1999 (Appendice, nr. 79); XII: 
I convegni «dispari», cioè "modernistici», di Acquasparta (ma, dei sei organizzati, gli ultimi due si svolsero a Gubbio e a Spoleto) divennero presto uno dei luoghi privilegiati del dibattito nazionale e internazionale fra i cultori della materia, favorendo l'incontro di alcuni dei testimoni superstiti della scuola desanctisiana e di tanti Maestri delle generazioni successive, italiani e stranieri ${ }^{31}$, con gli studiosi più giovani: del fervido clima di amicizia che regnava in quelle giornate si percepisce un'eco pure negli affettuosi ricordi o nelle dediche di questo o quel volume degli Atti a coloro che nel frattempo erano scomparsi (da Piero Treves a Mario Attilio Levi, da Mariella Cagnetta a Marcello Gigante) ${ }^{32}$.

Se quattro di tali riunioni furono dedicate a figure dominanti della storiografia tra Ottocento e Novecento (Beloch, 1986; Pais, 1992; Rostovzev, 1995; Momigliano, 1999), due affrontarono invece questioni riconducibili prevalentemente all'antichistica pre-unitaria, in una prospettiva generale, cioè italiana (convegno del 1988) e in un àmbito particolare, quello perugino (convegno del 1990). Emergeva così quell'interesse di Leandro Polverini anche per la tradizione «regionale» e «locale», di cui abbiamo tracce ulteriori, e significative, negli Atti della Deputazione di Storia Patria per l'Umbria $^{33}$ e in altri saggi ${ }^{34}$.

Fra gli esiti più ricchi e produttivi di questa molteplice esperienza segnalerei, accanto alle penetranti riletture dei lavori già editi, la scoperta, la pubblicazione e l'esegesi di materiali d'archivio di contenuto scientifico, di uso didattico, di testimonianza politica e di natura epistolare. Se le prime tre fattispecie riguardano in parti-

A. M. Biraschi, P. Desideri, S. Roda, G. Zecchini (a cura di), L'uso dei documenti nella storiografia antica, Gubbio, 22-24 maggio 2001, Napoli, 2003.

31 Dei primi furono presenti agl' Incontri perugini Mario Attilio Levi, Piero Treves e Luigi Moretti (mancarono invece Arnaldo Momigliano, scomparso nel 1987, cioè l'anno dopo il convegno inaugurale, e Silvio Accame, scomparso nel 1997: un accenno al tentato coinvolgimento di quest'ultimo in Appendice, nr. 45, p. 16, nota 52); dei secondi vengano ricordati, nell'ordine cronologico della loro partecipazione, Emilio Gabba, Ida Calabi Limentani, Marcello Gigante, Filippo Càssola e Lellia Cracco Ruggini.

32 P. Treves (1911-1992): Lo studio storico (Appendice, nr. 76), p. 17, nota 20; cf., inoltre, la dedica Alla memoria di Alessandra Gara e Piero Treves, in Maddoli (a cura di), L'Athenaion politeia di Aristotele 1891-1991 (nota 30), p. [3]. - M. A. Levi (1902-1998): Aspetti della storiografia di Ettore Pais (Appendice, nr. 78), p. [5]; MARCOnE (a cura di), Rostovtzeff e l'Italia (nota 30), p. [5] e 8. - M. Cagnetta (1950-1998): Aspetti della storiografia di Ettore Pais (Appendice, nr. 78), p. [5] e 330, nota 1; MARCONE (a cura di), Rostovtzeffe l'Italia (nota 30), p. [5] e 8. - M. Gigante (1923-2001): Arnaldo Momigliano nella storiografia del Novecento (Appendice, nr. 79), p. [VII] e 2, nota 3.

33 Appendice, nrr. 14 e 37.

34 L. Polverini, Fermo in età romana, in L. Polverini, N. F. Parise, S. Agostini, M. PAsquinucci (a cura di), Firmum Picenum, I, Pisa, 1987, p. 17-75, in particolare p. 19-23; Appendice, nrr. 26 e 38. 
colare i lasciti belochiano e desanctisiano ${ }^{35}$, l'ultima concerne in misura varia tutti gli archivi raggiunti dallo studioso in Italia (documenti belochiani, paisiani, desanctisiani, rostovzeviani e momiglianei di Roma e fraccariani di Roma e di Pavia), in Germania (materiali niebuhriani e meyeriani dell'Accademia delle Scienze di Berlino) e negli Stati Uniti (materiali ferreriani della Columbia University ed einsteiniani dell'Institute for Avanced Study di Princeton) ${ }^{36}$. Tali acquisizioni rimandano evidentemente ad alcuni presupposti: la mobilità del nostro Collega (ripetuti soggiorni di studio all'estero) ${ }^{37}$, le sue molteplici competenze linguistiche (estese anche al russo) ${ }^{38}$, un'attitudine irresistibile, se posso dir così, ad «intervistare» e a dialogare (donde la fruttuosità delle relazioni stabilite con i familiari dei suoi Auctores, da Margherita Beloch Piazzolla a Hans Eduard Meyer, settimo e ultimo figlio di Eduard, a Bogdan e Leo Raditsa, genero e nipote di Guglielmo Ferrero) ${ }^{39}$; del resto, la capacità di trarre il massimo profitto anche dalla «tradizione orale» risulta con evidenza da quanto ci ha trasmesso dei suoi colloqui di lavoro con Mario Attilio Levi, Arnaldo Momigliano, Silvio Accame, Piero Treves, Luigi Moretti e Karl Christ ${ }^{40}$.

Anche a volerla giudicare solamente negli aspetti «euristici», vale a dire, secondo la metafora niebuhriano-droyseniana, quale «arte del minatore» che "procura i mate-

35 Beloch: cf. supra, note 19 e 23. - De Sanctis: cf. supra, note 15, 16, 20. - Cf., inoltre, le Conversazioni sul Nazismo tenute da Momigliano a Radio Londra: Appendice, nr. 50, p. 172-178.

36 Beloch: cf. supra, note 19 e 23. Pais: Appendice, nr. 45, p. 17. De Sanctis: cf. supra, nota 23. Rostovzev: cf. supra, nota 23. Momigliano: Appendice, nr. 48. Fraccaro: Appendice, nrr. 15 e 29. - Niebuhr: Appendice, nrr. 18 e 36. Meyer: Appendice, nrr. 17, 19, p. 292-294, 28, 49. - Ferrero: Appendice, nr. 19. Einstein: Appendice, nr. 23 (con lettere di Francesco Ruffini, Guglielmo Ferrero, Albert Einstein, oltre che di Giuseppe Righetti, portavoce del ministro Alfredo Rocco).

37 Fra gli altri: 1969, Fondation Hardt, Genève; 1982, Akademie der Wissenschaften der DDR (Berlin); 1984-1985, Philipps-Universität Marburg; 1987, Fondation Hardt, Genève; 1989, Institute for Advanced Study (Princeton, NJ).

38 Anche dai viaggi a Mosca (1970), a Leningrado (1971), a Mosca (1973) dipendono i contributi, ricchi di notizie fino ad allora poco note, sulla fortuna di Beloch e Meyer in Russia: Appendice, nrr. 11 e 17, p. 213-215.

39 Margherita Beloch Piazzolla: cf. supra, nota 18. - Hans Eduard Meyer: Appendice, nr. 17, p. 199-200, nota 1. - Bogdan e Leo Raditsa: Appendice, nrr. 19, p. 293, nota 56 e 23, p. 268, nota 3.

40 Accame: Appendice, nr. 8, p. XXIII, nota 1. - Moretti: Appendice, nr. 8, p. XXVI, nota 10. - Levi, Momigliano, Accame, Treves, Moretti: Appendice, nr. 13, passim. - Levi: Appendice, nrr. 44, p. 154 e 45, p. 11, nota 19. - Momigliano: Appendice, nrr. 48, p. 4 e 50, p. 168. - Treves: Appendice, nr. 45, p. 11, nota 19. - Christ: Appendice, nr. 50, p. 163. - A questi bisogna aggiungere i contatti personali con la Scuola di Pavia, in particolare con Albino Garzetti ed Emilio Gabba. Cf., inoltre, Appendice, nr. 15, p. 68, * e 74 , nota 23 . 
riali ${ }^{41}$ ", l'attività di Leandro Polverini come storico della storiografia moderna sul mondo antico non ha dunque mai corrisposto a quello che Arnaldo Momigliano chiamava «un passatempo domenicale ${ }^{42}$ ». Oggi, anzi, possiamo rilevare come l'impegno editoriale del Collega rispetto alla produzione scientifica di alcuni Maestri ${ }^{43}$ abbia trovato seguaci non meno agguerriti sotto il profilo «tecnico» e filologico (penso, ad esempio, alla "Nuova edizione» della Storia economica e sociale dell'Impero romano di Michele Rostovzev, curata da Arnaldo Marcone) ${ }^{44}$; e come l'espressione di tale impegno che valorizza, per citare una formula di Corinne Bonnet e Véronique Krings, "l'apport des correspondances à l'histoire des travaux scientifiques ${ }^{45}$ ", continui ad arricchirsi di nuovi contributi (rimanendo nel campo di nostra competenza, segnalo i recenti lavori di Marcella Guglielmo sul carteggio De Sanctis-Fraccaroli e di Valentina Cuomo sul carteggio Wilamowitz-De Sanctis) ${ }^{46}$.

A questo riconoscimento deve però seguire un altro, più significativo ancora. Leandro Polverini ha interpretato nel migliore dei modi l'assioma di Arnaldo

41 Cito da J. G. Droysen, Sommario di Istorica, Traduzione e nota di D. Cantimori, Firenze, 1943, p. 18 (un giudizio polveriniano sulla terza edizione dell'opera in Appendice, nr. 3).

42 A. Momigliano, Recensione di H. Berve, Storia greca, Bari, 1959, p. 665-672 (a p. 672 la citazione) $=$ Terzo contributo alla storia degli studi classici e del mondo antico, Roma, 1966, p. 699-708 (a p. 708 la citazione).

43 Cf. supra, note 15, 16, 20.

44 M. RostovtzefF, Storia economica e sociale dell'Impero romano, Nuova edizione accresciuta di testi inediti, a cura di A. Marcone, Milano-Firenze, 2003. - Delle iniziative precedenti voglio citare almeno quelle di segno belochiano: alcuni capitoli della Wirtschaftsgeschichte Athens, a cura C. Ampolo, in Opus 4 (1985), p. 9-28 e in Polverini (a cura di), Aspetti della storiografia di Giulio Beloch, p. 79-104; Campania. Storia e topografia della Napoli antica e dei suoi dintorni, a cura di C. Ferone e F. Pugliese Carratelli, Napoli, 1989 (cfr. Appendice, nr. 20, p. 14, nota 13); Surrentum im Alterthum / Sorrento nell'antichità, hrsg. von / a cura di A. Russi, San Severo (FG), 1993; Storia della popolazione d'Italia, Introduzione di L. Del Panta e E. Sonnino, Firenze, 1994. Quest'ultimo volume realizza, in qualche modo, un auspicio formulato da Leandro Polverini (Appendice, nr. 20, p. 17-18).

45 C. Bonnet, V. Krings, "S'écrire et écrire sur l'Antiquité. L'apport des correspondances à l'histoire des travaux scientifiques. Un chantier en cours", Quaderni di Storia 32 (2006), 63, p. 151-185 (il medesimo numero della rivista comprende anche altri lavori sull'argomento); C. Bonnet, V. KRINGs (éd.), S'écrire et écrire sur l'Antiquité. L'apport des correspondances à l'histoire des travaux scientifiques, Grenoble, 2008 (dove compare anche il saggio corrispondente ad Appendice, nr. 49).

46 M. Guglielmo (a cura di), Il carteggio Gaetano De Sanctis-Giuseppe Fraccaroli, Firenze, 2007; V. Cuomo, "Il carteggio di Ulrich von Wilamowitz-Moellendorff e Gaetano De Sanctis", Quaderni di Storia 34 (2008), 68, p. 165-183. - Nella suddetta prospettiva cf., inoltre, G. Granata (a cura di), L'archivio Arnaldo Momigliano: inventario analitico, Prefazione di R. Di Donato, Roma, 2006. 
Momigliano secondo cui «the only justification for the history of scholarship is the promotion of scholarship itself ${ }^{47}$ »: una citazione più volte riproposta dal Collega ${ }^{48}$.

L'adesione a tale principio ha, nella sua opera scientifica, degli esiti che possiamo cogliere su vari piani.

Per quanto riguarda la cultura specialistica, cioè, nel caso particolare, la conoscenza della storia romana in senso "tecnico», segnalo anzitutto quelle che appaiono delle riprese di motivi desanctisiani. Credo, infatti, che uno dei giudizi espressi nel paragrafo La più grande Roma dell'ampio saggio derivato dalla tesi di laurea - «la conclusione del processo di romanizzazione delle genti italiche non aveva portato alla formazione d'uno stato, per così dire, nazionale ${ }^{49}{ }$ - discenda, in ultima istanza, da note formulazioni del suo Auctor maggiore sull' «italicizzazione» mancata della penisola dopo la guerra sociale, che sarebbero divenute oggetto di penetranti esegesi nei contributi polveriniani di storia della storiografia ${ }^{50}$; e che radici non diverse abbia, in un altro dei primi lavori, tratto dalla tesi di specializzazione, il riconoscimento che «il difetto di fondo della civiltà antica [era] il suo carattere d'élite e la sua scarsa penetrazione nella massa ${ }^{51}$ ", debitore con tutta evidenza della replica di Gaetano De Sanctis a Michele Rostovzev nel memorabile confronto a proposito della Social and Economic History of the Roman Empire, anch'essa richiamata dagli scritti posteriori del Collega ${ }^{52}$.

Nella tradizione risalente a uno dei filoni più ricchi delle scuole belochiana e fraccariana è invece collocabile, anche per alcune sue dichiarazioni esplicite, l'interesse per la storia «locale» e «regionale», preannunciato da un intervento nel Convegno

47 "New Paths of Classicism in the Nineteenth Century", History and Theory 21 (1982), 4, Beiheft 21, p. 1 (1'“Introduction" al saggio, p. 1-2, non compare nella tr. it., Nuovi sentieri del Classicismo nel XIX secolo, in A. MomiglianO, Tra storia e storicismo, Pisa, 1985, p. 99-192).

48 Appendice, nrr. 19, p. 298, nota 78 e 20, p. 18, nota 38.

49 "L'aspetto sociale del passaggio dalla Repubblica al Principato" (cf. supra, nota 7), p. 439.

50 De SANCTIS, La guerra sociale (Appendice, nr. 74), p. 41 («Non si trattava più per essi di romanizzare l'Italia, ma di creare un'Italia degli Italiani: alto ideale che nacque e tramontò con la guerra detta sociale o marsica...»): cf. Appendice, nr. 8, p. XXXV-XXXVI.

51 "Le città dell'Impero nell'epistolario di Plinio" (cf. supra, nota 7), p. 220.

52 G. De Sanctis, Recensione di M. Rostovtzew, The Social and Economic History of the Roman Empire, Oxford, 1926, Rivista di Filologia e di Istruzione Classica n. s. 4 (1926), 4, p. 553 = Scritti minori, VI, 1, p. 312 ("Lo sforzo livellatore in cui la civiltà classica si consumò e decadde non fu quello della penetrazione nelle masse dei popoli che l'avevano creata. Fu quello della sua imposizione violenta ai popoli che n'erano al di fuori»); G. DE SAnctis, "Presentazione", in M. RostovZeV, Storia economica e sociale dell'Impero romano, Firenze, 1933, p. VI («... la civiltà dell'Impero è stata eminentemente aristocratica, e non ha fatto mai vera prova di penetrazione nelle masse»): cf., però, le osservazioni al riguardo in Appendice, nr. 5, p. 1061-1062. 
in memoria di Plinio Fraccaro ${ }^{53}$ e concretizzatosi pienamente nei lavori su Firmum Picenum e sulle Regiones augustee ${ }^{54}$.

Passando al tema centrale del nostro discorso, collocherei tra gli apporti maggiori di Leandro Polverini al molteplice dibattito svoltosi a partire dagli Anni Settanta nel campo della storia della storiografia moderna sul mondo antico la ricostruzione delle prime fasi e della successiva crescita di quelle che vengono da lui considerate le istituzioni dominanti nell'àmbito nazionale fra Ottocento e Novecento: da un lato la Scuola romana di Giulio Beloch, «rinnovatore» o «rifondatore degli studi di storia antica in Italia ${ }^{55}$ ", dall'altro la Scuola torinese (poi romana) di Gaetano De Sanctis e la Scuola pavese di Plinio Fraccaro ${ }^{56}$.

È inutile ribadire l'importanza delle nuove o prime edizioni di lavori propriamente storiografici, come le recensioni e il frammento sulla guerra sociale di Gaetano De Sanctis, o della pubblicazione di tante altre pagine di contenuto didattico e progettuale, come i testi relativi alla prima lezione e ad alcuni corsi tenuti da Giulio Beloch ${ }^{57}$ o il Programma della «rivista storica che non fu mai fondata» e gli Elementi per un promemoria sull'utilità d'un insegnamento di metodologia e storia della scienza dell'antichità dovuti al suo maggiore allievo ${ }^{58}$. Per quanto riguarda gli scambi epistolari, dalle ricerche del Collega è derivata la nostra possibilità di conoscere meglio, al di là delle circostanze accademiche o personali che, talvolta, ne sono state l'occasione, la libertà di giudizio e la tensione morale e civile dei dialoghi, continuati per decenni, fra Beloch

53 L. Polverini, "Di un tema metrico attestato in epigrafi della Transpadana", in Convegno in memoria di Plinio Fraccaro, Pavia, 8-10 settembre 1975, Athenaeum, Fascicolo speciale (1976), p. 145-151 (p. 151: «'impone... la precisazione concettuale e storica di termini come cultura "locale" o "regionale" e del loro rapporto, necessariamente bivalente, con quella che solo per comodità si è definita cultura "ufficiale" "). - In merito alla sensibilità di un allievo di Plinio Fraccaro, cioè Albino Garzetti, per la storia locale (donde una possibile influenza diretta di quest'ultimo sul proprio discepolo) cf. le notizie riportate in Appendice, nr. 42, p. 23-24. Inoltre: Appendice, nr. 43, p. 254.

54 Polverini, "Fermo in età romana", p. 19-23. Cf., inoltre, Appendice, nrr. 14, 26, 37, 38. - L. Polverini, "Le regioni nell'Italia romana”, Geographia antiqua 7 (1998), p. 23-33. Cf., inoltre, L. PolverinI, "Errata corrige (con un auspicio)", Geographia antiqua 8-9 (1999-2000), p. 134.

55 Appendice, nrr. 6, p. 153, 9, p. 1370, 20, p. 16, 47, p. 8.

56 «... le due personalità più vigorose e significative che la storia antica abbia avuto in Italia nella prima metà del nostro secolo... accomunate da questa circostanza e dal fatto di aver dato vita alle due più importanti scuole italiane di storia antica»: Appendice, nr. 15, p. 69. - Per un bilancio relativo alla seconda scuola cf., ora, le vivide pagine di E. GABBA, "Sull'insegnamento di Plinio Fraccaro all'Università di Pavia. Ritratti di maestro e allievi", Athenaeum 97 (2009), 1, p. 229-246. Inoltre: E. GABBA, Conversazione sulla storia, a cura di U. Laffi, Pisa-Cagliari, 2009, passim.

57 Cf. supra, note 19 e 23 (Appendice, nrr. 9 e 24).

58 Cf. supra, nota 20 (Appendice, nrr. 7 e 13). 
e De Sanctis ${ }^{59}$ e, soprattutto, fra De Sanctis e Fraccaro e De Sanctis e Momigliano ${ }^{60}$ (insieme con l'altezza dei ragionamenti storici e la varietà e ricchezza delle proposte interpretative, che anticipano o commentano o precisano quanto destinato alle pubblicazioni scientifiche di quei Maestri). Caratteri non diversi presentano, del resto, anche gli epistolari con interlocutori «esterni»: quelli fra Beloch e Meyer ${ }^{61}$ e fra De Sanctis e Rostovzev ${ }^{62}$.

Quanto finora messo in evidenza riguarda l'opera di alcuni protagonisti. Gaetano De Sanctis e Michele Rostovzev, "con Eduard Meyer», sono, a giudizio di Leandro

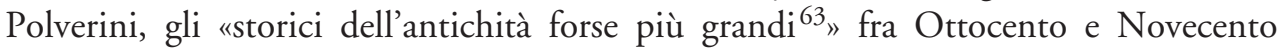
(una convinzione ribadita pure in altra forma: «De Sanctis e Rostovzev ci appaiono rispettivamente - l'ultimo grande storico dell'Ottocento e il primo grande storico del Novecento ${ }^{64}$ ). Pur non collocandosi a livelli di eccellenza paragonabili ai precedenti, anche una figura come quella di Ettore Pais, che Gaetano De Sanctis posponeva comunque, tra i discepoli di Theodor Mommsen, a Ettore De Ruggiero ${ }^{65}$, rientra nelle alte sfere dell'Altertumswissenschaft del tempo: donde la posizione autonoma riconosciutale in uno degl'Incontri di Acquasparta ${ }^{66}$.

Ma l'attenzione del Collega per la storia "locale" o "regionale"”, già messa in rilievo, lo ha portato a studiare con uguale interesse personaggi minori dal punto di vista dei parametri scientifici più aggiornati della nuova Methode germanica e tuttavia degni di nota in funzione di una storia dell'antichistica italiana pre-unitaria e immediatamente post-unitaria, che miri da un lato a riconoscere $\mathrm{i}$ loro contributi specifici di raccoglitori, editori e valorizzatori di materiali con un forte radicamento nel territorio, dall'altro a precisare la collocazione di essi nel quadro culturale e politico delle società che li esprimevano. Il tentativo di gettare le basi di quella che, dionisottianamente,

59 Cf. supra, nota 23 (Appendice, nr. 5).

60 De Sanctis-Fraccaro: cf. supra, nota 23 (Appendice, nrr. 15 e 29). Altri corrispondenti di Fraccaro: Appendice, nr. 29, p. 419-422 (Ettore Pais), 422-424 (Carlo Pascal), 424-427 (Giorgio Pasquali), 427-429 (Eduard Schwartz). - De Sanctis-Momigliano: cf. supra, nota 23 (Appendice nr. 48).

61 Cf. supra, nota 23 (Appendice, nrr. 17, 28, 49).

62 Cf. supra, nota 23 (Appendice, nr. 40).

63 Appendice, nr. 5, p. 1060.

64 Appendice, nr. 40, p. 99.

65 G. De Sanctis, ["Epigrafisti e cultori dell'antichità di recente scomparsi: P. Foucart, B. Haussoullier, E. De Ruggiero, E. Costa”], Rivista di Filologia e di Istruzione Classica n. s., 4 (1926), 4, p. 571-572 = Scritti minori, VI, 2, p. 745. - Sull'Autore (1839-1926) in generale: M. ElEFANTE, "Ettore De Ruggiero”, in La cultura classica a Napoli nell'Ottocento, Premessa di M. Gigante, Napoli, 1987, II, p. 727-754; M. Elefante, "De Ruggiero, Ettore”, in Dizionario Biografico degli Italiani, XXXIX, Roma, 1991, p. 244-248.

66 Appendice, nr. 78 (dove Appendice, nr. 45). Cf. ibid., p. 327-339, il magistrale "Bilancio conclusivo" di Filippo Càssola. 
denominerei una geografia e storia dell'antiquaria italiana ${ }^{67}$ aveva il suo modello di riferimento nei capitoli "pre-unitari» e "post-unitari» delle due monografie pubblicate all'inizio degli Anni Sessanta da Piero Treves, che avrebbe continuato a esplorare gli àmbiti suddetti fino alla morte, raccogliendo una parte delle sue ricerche in Tradizione classica e rinnovamento della storiografia e in Ottocento italiano fra il nuovo e l'antico ${ }^{68}$ : nella medesima prospettiva del Maestro, ma con il consueto valore aggiunto di un ampio utilizzo dei documenti d'archivio, Leandro Polverini ha promosso da una parte nuove ricognizioni sistematiche (per quanto, dichiaratamente, non esaustive) della materia nel III degl' Incontri perugini, dedicato allo studio storico del mondo antico nella cultura italiana dell'Ottocento ${ }^{69}$, dall'altra l'analisi di un contesto particolare nel V, incentrato sull'erudizione e l'antiquaria perugina del medesimo periodo ${ }^{70}$. La formula del primo di questi due convegni di Acquasparta, in cui la suddivisione per discipline (filologia classica, etruscologia, linguistica, filosofia, diritto, epigrafia, numismatica, papirologia, antiquaria, archeologia classica, antichità cristiane, storia orientale, storia greca, storia romana) coesisteva non di rado con l'attenzione per i diversi àmbiti geografico-politici (Lombardo-Veneto, Stato Pontificio, Regno di Napoli), offriva un modello a progetti futuri di sintesi; cui l'Incontro perugino di argomento «locale» somministrava la sua parte di Realien, dando nel contempo un esempio di ricerca «mirata». Che quest'ultimo indirizzo sia molto produttivo risulta ormai da numerose pubblicazioni, precedenti, contemporanee e successive, tra le quali voglio ricordare almeno quelle realizzate, con

67 C. Dionisotti, Geografia e storia della letteratura italiana, Torino, 1967 (edizioni e ristampe successive).

68 P. Treves, L'idea di Roma e la cultura italiana del secolo XIX, Milano-Napoli, 1962; ID. (a cura di), Lo studio dell'antichità classica nell'Ottocento, Milano-Napoli, 1962. - ID., Tradizione classica e rinnovamento della storiografia, Milano-Napoli, 1992; ID., Ottocento italiano fra il nuovo e l'antico, I, Alle prese con la storia, II, Regioni, III, Le tre corone?..., Modena, 1992. - Inoltre: ID., "Gli studii classici nell'Italia del Novecento", Annali dell'Istituto Italiano per gli Studi Storici 12 (1991-1994), Studi per Ettore Lepore, p. 624-638; ID., Scritti novecenteschi, a cura di A. Cavaglion e S. Gerbi, Bologna, 2006. - Una rassegna completa delle ultime pubblicazioni dell'Autore in C. FranCo (a cura di), Piero Treves dal 1930 al 1996, Istituto Universitario Orientale, Bibliografie, 5, Napoli, 1997.

69 Appendice, nr. 76 (dove Appendice, nr. 27: a p. 16-17, nota 18 un rinvio a contributi analoghi di Ettore Lepore, Francesco Casavola e Marcello Gigante). Cf., da ultimo, alcuni dei saggi raccolti in E. GABBA, Riflessioni storiografiche sul mondo antico, Como, 2007.

70 Appendice, nr. 77 (dove Appendice, nrr. 34 e 35). Dei tre personaggi analizzati nel secondo contributo di Leandro Polverini (Appendice, nr. 35) almeno Ariodante Fabretti (1816-1894) è tutt'altro che un «minore». 
i loro collaboratori, da Marcello Gigante e da Arnaldo Marcone, aventi per oggetto gli studi classici a Napoli ${ }^{71}$ e nelle Venezie ${ }^{72}$.

Uno dei tratti più significativi delle ricognizioni storiografiche di Leandro Polverini, al di là dell'apprezzamento degli obiettivi propriamente scientifici conseguiti, a giudizio dello Studioso, dai suoi Auctores $^{73}$ (e che non è possibile qui nemmeno elencare), mi sembra la ricorrente individuazione degli aspetti dell'opera loro che inverano l'assioma crociano secondo cui «ogni vera storia è storia contemporanea ${ }^{74} »$ : donde, per esempio, l'attenzione alle ricadute storiografiche del razzismo e dell'antisemitismo di Giulio Beloch ${ }^{75}$ o delle prospettive «risorgimentali» e «statutarie» e delle opzioni anticapitalistiche e sociali (vicine a quelle del Partito Popolare, in cui militò) di Gaetano De Sanctis ${ }^{76}$; ma spunti analoghi riguardano anche l'opera di Eduard Meyer e di Arnaldo Momigliano.

La questione del rapporto fra storiografia e politica diventa cruciale durante il ventennio fascista, in particolare dopo il 1931, anno dell'imposizione ai docenti universitari del giuramento di fedeltà. La scelta di Gaetano De Sanctis di respingerlo (il Maestro fu della piccola schiera di professori, 12 su oltre 1200, ch'ebbero tale coraggio) ${ }^{77}$ non trovò imitatori fra i classicisti legati a lui da vincoli di stima o di scuola, nemmeno

71 La cultura classica a Napoli nell'Ottocento, Premessa di M. GiganTe, I, II, Napoli, 1987; M. Capasso, S. Cerasuolo, M. L. Chirico, G. Giannantoni, M. Gigante, F. Giordano, E. Paratore, Ar. Salvatore (a cura di), Momenti della storia degli studi classici fra Ottocento e Novecento, Atti del Seminario (Napoli, 27-28 febbraio 1986), Premessa di M. Gigante, Napoli, 1987; La cultura classica a Napoli nell'Ottocento. Secondo contributo, Premessa di M. Gigante, Napoli, 1991.

72 M. BuOra, A. MARCONE (a cura di), La ricerca antiquaria nell'Italia nordorientale dalla Repubblica Veneta all'Unità, Antichità Altoadriatiche, 64, Trieste, 2007; A. BuOnOpane, M. BuOra, A. Marcone (a cura di), La ricerca epigrafica e antiquaria nelle Venezie dall'età napoleonica all'Unità, [Atti dell'Incontro di Studio (Udine-San Daniele del Friuli, 6-7 ottobre 2006)], Firenze, 2007.

73 In base al criterio dell" «efficacia... esercitata nel prosieguo degli studi»: Appendice, nr. 15, p. 68.

74 B. Croce, Teoria e storia della storiografia, Bari, 1917, [Capitolo] I, Storia e cronaca: decima edizione, Bari, 1973, p. 4.

75 Appendice, nr. 17, p. 206, nota 16.

76 Appendice, nrr. 8, p. XXXV-XXXVII, 13, p. 458-462, 40, p. 103-109.

77 Appendice, nr. 23, p. 277, nota 42. Circa il problema in generale, da ultimo: H. GoETz, Il giuramento rifiutato. I docenti universitari e il regime fascista, Firenze, 2000; G. BOATTI, Preferirei di no. Le storie dei dodici professori che si opposero a Mussolini, Torino, 2001. Documenti epistolari delle reazioni suscitate dall'atto di coraggio desanctisiano - per il quale cf., anzitutto, Ricordi, p. 143-157 (notizie autobiografiche) e p. 236-255 (lettere di Gaetano De Sanctis, Umberto Zanotti Bianco, Giorgio Levi Della Vida, Guglielmo Ferrero, Hugh Last, Michel Lhéritier, Maurice Holleaux, Frank E. Adcock, Gustave Glotz, Norman H. Baynes, Tenney Frank) - in Appendice, nrr. 15, p. 77-78 (Plinio Fraccaro) e 40, p. 110 (Michele Rostovzev). 
in quelli anti-fascisti o a-fascisti, come Plinio Fraccaro e Arnaldo Momigliano ${ }^{78}$. Leandro Polverini mette in luce lo sforzo di quest'ultimo di trovare uno spazio, ridotto ma effettivo, di autonomia di giudizio: nella Prolusione torinese del 1936, a parte le concessioni all" "ora, solenne, che volge[va] per la patria ${ }^{79}$ ", «la scelta del tema, sulla trasformazione del concetto di pace nel mondo greco e romano, era coraggiosa sia dal punto di vista accademico, sia dal punto di vista politico. Riaccorpava, infatti, la storia greca e la storia romana, che erano state appena scisse nel titolo della cattedra torinese in funzione dell'ormai imperante filoromanesimo; e, soprattutto, contrapponeva con forza l'ideale della pace alla esaltazione bellicistica dell'epoca ${ }^{80}$ \%. Quanto invece alla «maggioranza [che] accettò (per convinzione, per opportunismo, per viltà) forme varie di compromesso con il regime, al quale alcuni aderirono pienamente», il giudizio del Collega è drastico: «Dichiarando (e forse credendo) di fare storia fascista, in realtà essi [collocarono] in una cornice fascista la storia che avrebbero comunque fatto ${ }^{81}$ ». Un'opinione vivacemente discussa, e ancora discutibile ${ }^{82}$.

Nell'esaminare l'opera di alcuni degli antichisti più «consenzienti», sulla fine o dal principio, al despota egli abbandona talvolta il distacco e la misura che caratterizzano, in genere, le sue valutazioni: circa l'ultima, e discendente, parabola di Ettore Pais ripete un icastico giudizio di Piero Treves ${ }^{83}$; mentre «il rinnovato confronto fra Cesare e Mussolini» proposto nei Due imperi di Roma di Luigi Pareti gli sembra «vacua - e indegna - retorica, antitesi della concezione storiografica, sobria e fattuale, che [l'autore] aveva mutuato dai suoi maestri [De Sanctis e Beloch $]^{84}$.

78 La solidarietà espressagli dal primo (Appendice, nr. 15, p. 77-78) non gli fu pretesto per autoassolversi dalla colpa di aver fatto parte della maggioranza di coloro che "dovettero, per vivere, piegare la testa e farsi spergiuri»: P. FRACCARO, Relazioni e discorsi degli anni del rettorato (1945-1959), Fonti e studi per la storia dell'Università di Pavia, 6, Milano, 1983, p. 16.

79 "Prolusione" (Università di Torino, dicembre 1936), in C. DionisotTi, Ricordo di Arnaldo Momigliano, Bologna, 1989, p. 109-130 (a p. 109 la citazione), ripubblicata, con il titolo Koinè Eiréne, Pax Romana, Pax Christiana, in A. Momigliano, Nono contributo alla storia degli studi classici e del mondo antico, a cura di R. Di DonATO, Roma, 1992, p. 409-423 (a p. 409 la citazione). Per l'annotazione del 1982, in cui l'Autore si autodefinisce «ebreo e non fascista», cf. DionisotTi, Ricordo, p. 97, discusso in Appendice, nr. 50, p. 166.

80 Appendice, nr. 50, p. 167.

81 Appendice, nr. 44, p. 160.

82 Ibid., nota 59.

83 Lo studio dell'antichità classica nell'Ottocento, p. 1162 («Meglio, perciò, gli sarebbe convenuto il silenzio, invece di un triste trentennio di battaglie accademiche e di vacue scritture praticistiche»), discusso in Appendice, nr. 45, p. 16. Cf., del resto, le osservazioni già formulate in Appendice, nr. 39, p. 439-440.

84 Appendice, nr. 44, p. 152. - Nel saggio viene ripubblicata (p. 162-163) la recensione che al volume paretiano dedicò il ventiduenne Santo Mazzarino (Il Popolo di Sicilia, 3 maggio 1938). 
In altri termini, Leandro Polverini, figlio di una vittima delle rappresaglie tedesche ${ }^{85}$, può ammettere che «altro è il giudizio politico e umano, altro è il giudizio storiografico (anche se la nostra formazione classica ci fa tutti un po' tacitiani) ${ }^{86}{ }_{\| ;}$ma, pure «in tempi di revisionismo ${ }^{87}$ ", non sembra incline ad assecondare la "nouvelle vague»: una scelta, quest'ultima, dalla quale farei dipendere anche, nei lavori dedicati alle Regiones augustee, il giudizio critico sul tentativo leghista di riconoscere nella cultura celtica le radici della cosiddetta Padania, dove, in realtà, «le popolazioni celtiche

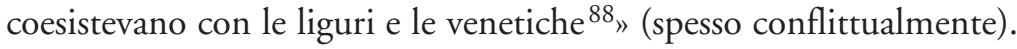

Per quanto riguarda la presenza di una dimensione "politica» nell'opera dello Studioso, voglio accennare, infine, alle preoccupazioni da lui espresse circa uno dei fondamenti del nostro lavoro: la conoscenza delle due lingue classiche. Il venir meno di tale condicio sine qua non - intravisto dal Collega fin dagli Anni Settanta nella recensione di un volume francese ${ }^{89}$ ( $m a$ il fenomeno coinvolge da tempo anche la storiografia di àmbito anglosassone $)^{90}$ - riguarda oggi pure la formazione antichistica impartita nell'Università italiana. Sulla gravità di questo fenomeno la comunione di giudizio con Filippo Càssola era completa, e mi piace notarlo. Rimane aperta la questione se nello sfascio istituzionale e culturale del nostro Paese un'inversione di tendenza sia possibile.

Da quando è un professore universitario fuori ruolo ${ }^{91}$, e dunque (relativamente) libero, come disse una volta, «dai sempre più pletorici e invadenti impegni della vita accademica ${ }^{92} "$, Leandro Polverini ha continuato le sue ricerche, nelle quali mantiene tuttora uno spazio preponderante la storiografia moderna sul mondo antico.

Agli ultimi lavori di tale argomento ${ }^{93}$ ci attendiamo che faccia séguito la preannunciata edizione integrale degli epistolari Beloch-Mayer, De Sanctis-Rostovzev e De

85 L. Polverini, Foreword, in M. G. NASH, The Price of Innocence, [Knutsford], 2005, p. IX-X = Prefazione (all'edizione inglese), in Il prezzo dell'innocenza, Edizione italiana a cura di E. Polverini, Prefazione di I. Ferri, Cavriglia, 2009, p. XI-XII.

86 Appendice, nr. 44, p. 160.

87 Ibid.: «In tempi di revisionismo bisogna esser chiari».

88 Appendice, nr. 37, p. 76-77.

89 Nota su M. BÉnabou, La résistance africaine à la romanisation, Paris, 1976, Athenaeum n. s., 56 (1978), 1-2, p. 185-190, in particolare p. 189.

90 Michele Rostovzev lo segnalava già negli Anni Venti per le Università degli Stati Uniti: Appendice, nr. 40, p. 109. Sul problema, da ultimo: G. BANDELli, Recensione di J. Briscoe, A Commentary on Livy. Books 38-40, Oxford, 2008, in corso di stampa su Athenaeum 99 (2011).

$91 \quad 1^{\circ}$ novembre 2008.

92 Traggo la formula dal suo atto di omaggio a Filippo Càssola e Ruggero Fauro Rossi: Appendice, nr. 43, p. 249.

93 Per quelli relativi al 2009-2010 cf. Appendice, nrr. 50-55. 
Sanctis-Momigliano ${ }^{94}$. Ma la conoscenza che il Collega e Amico ha degli archivi cui essi appartengono, e di tante altre cose, fa sperare anche in ricognizioni foriere di nuove scoperte e di nuovi studi. Egli ha da insegnarci ancora molto.

\begin{tabular}{l} 
Gino BANDELlI \\
\hline Università degli Studi di Trieste \\
Via del Lazzaretto Vecchio 6 \\
34123 Trieste \\
Italia \\
bandelgi@units.it
\end{tabular}

\section{Appendice}

Contributi di Leandro Polverini

alla storia della storiografia moderna sul mondo antico

(1967-2010)

\section{Saggi}

1. "Omaggio a Croce - vent'anni dopo", Vita e Pensiero 50 (1967), 3, p. 320-321.

2. "Che cos'è la storia?", Vita e Pensiero 50 (1967), 5, p. 526-530.

3. "Il Sommario del Droysen: cent'anni dopo", Vita e Pensiero 51 (1968), 12, p. 10071012.

4. "Avvertenza", in G. De Sanctis, Scritti minori, Novamente editi da A. Ferrabino e

S. ACCAME, VI, Recensioni - Cronache e commenti, 1, Roma, 1972, p. 7-8.

5. "Gaetano De Sanctis recensore", Annali della Scuola Normale Superiore di Pisa s. III, 3 (1973), 4, p. 1047-1094.

6. "Una lettera di Giovanni Pascoli a Giulio Beloch", Aevum 48 (1974), 1-2, p. $153-154$.

7. "Programma di una rivista storica che non fu mai fondata", Annali della Scuola Normale Superiore di Pisa s. III, 5 (1975), 1, p. 421-447.

8. "Introduzione", in G. De SANCTIS, La guerra sociale, Opera inedita, a cura di L. PolverinI, Firenze, 1976, p. XIII-XLIII.

9. "La prima lezione di Giulio Beloch alla Sapienza", Annali della Scuola Normale Superiore di Pisa s. III, 7 (1977), 4, p. 1369-1388.

94 Beloch-Meyer: Appendice, nrr. 17, p. 199, 218 e 219, 28, p. 243, 49, p. 105 e 111. De Sanctis-Rostovzev: Appendice, nr. 40, p. 98 e 102. De Sanctis-Momigliano: Appendice, nr. 48, p. 30. 
10. "Bibliografia degli scritti di Giulio Beloch", Annali della Scuola Normale Superiore di Pisa s. III, 9 (1979), 4, p. 1429-1462.

11. "Di una traduzione russa della Griechische Geschichte del Beloch", Critica storica 16 (1979), 4, p. 521-544.

12. "Bibliografia degli scritti di Giulio Beloch. Primo addendum", Annali della Scuola Normale Superiore di Pisa s. III, 11 (1981), 3, p. 825-827.

13. "La Storia dei Romani che non fu scritta", Studi romani 30 (1982), 4, p. 449-462.

14. "[Bibliografia e archivistica nella ricerca storica in Umbria], Incontro di studio (Spoleto, 25 ottobre 1981)", Bollettino della Deputazione di Storia Patria per l'Umbria 81 (1984), p. 282-283 e p. 296-298.

15. "Fraccaro e De Sanctis", Athenaeum n. s. 63 (1985), 1-2, p. 68-113.

16. "Karl Christ fra storia e storiografia", Storia della storiografia 5 (1986), 9, p. 146-152.

17. "Il carteggio Beloch-Meyer", in K. Christ, A. Momigliano (a cura di / hrsg. von), L'antichità nell'Ottocento in Italia e Germania / Die Antike im 19. Jahrhundert in Italien und Deutschland, Atti della settimana di studio / Akten der Studienwoche (Trento, 1-5 settembre / Trento, 1.-5. September 1986), Bologna / Berlin, 1988, p. 199-219.

18. "Lettere di Giacomo Leopardi a B. G. Niebuhr", Rivista Storica Italiana 100 (1988), 1, p. 220-233.

19. "Cesare e Augusto nell'opera storica di Guglielmo Ferrero", in K. ChrIST, E. GABBA (hrsg. von), Römische Geschichte und Zeitgeschichte in der deutschen und italienischen Altertumswissenschaft während des 19. und 20. Jahrhunderts, I, Caesar und Augustus, [Atti del Convegno (Marburg, 4-9 settembre 1988)], Como, 1989, p. 277-298.

20. "Introduzione", in L. Polverini (a cura di), Aspetti della storiografia di Giulio Beloch, [Atti del Convegno (Acquasparta, 19-21 maggio 1986)], Incontri perugini di storia della storiografia antica e sul mondo antico, I, Napoli, 1990, p. 9-18.

21. "Morcelli e Winckelmann", in I. Calabi Limentani (a cura di), Stefano Antonio Morcelli 1737-1821, Atti del Colloquio (Milano-Chiari, 2-3 ottobre 1987), Brescia, 1990, p. 117-129.

22. "Arnaldo Momigliano", Nuova Secondaria 8 (1990-1991), 10, p. 79-81.

23. "Albert Einstein e il giuramento fascista del 1931", Rivista Storica Italiana 103 (1991), 1, p. 268-280.

24. "Il primo insegnamento di 'Geografia antica' in Italia", Geographia antiqua 1 (1992), p. 5-14.

25. "L'Istituto Italiano per la Storia Antica", in P. Vian (a cura di), Speculum mundi. Roma centro internazionale di ricerche umanistiche, Roma, 1992 [ristampa anastatica: Roma, 1993], p. 584-596.

26. "Prefazione", in M. C. Spadoni Cerroni, A. M. Reggiani Massarini, Reate, Pisa, 1992, p. 7-8.

27. "Introduzione", in L. Polverini (a cura di), Lo studio storico del mondo antico nella cultura italiana dell'Ottocento, [Atti del Convegno (Acquasparta, 30 maggio - 
$1^{\circ}$ giugno 1988)], Incontri perugini di storia della storiografia antica e sul mondo antico, III, Napoli, 1993, p. 9-17.

28. "L'Athenaion politeia nel carteggio Beloch-Meyer", in G. MAdDoli (a cura di), L'Athenaion politeia di Aristotele 1891-1991. Per un bilancio di cento anni di studi, [Atti del Convegno (Acquasparta, 27-29 maggio 1991)], Incontri perugini di storia della storiografia antica e sul mondo antico, VI, Napoli, 1994, p. 241-251.

29. "Dal carteggio di Plinio Fraccaro", Athenaeum n. s. 83 (1995), 2, p. 411-429.

30. "La 'Scuola' di Via Milano", in P. VIAN (a cura di), "Hospes eras, civem te feci». Italiani e non Italiani a Roma nell'ambito delle ricerche umanistiche, Roma, 1996, p. 109-111.

31. "L'opera di Pavan tra classicità e cristianesimo", Lettera dall'Italia 11 (1996), 41, p. 46.

32. "La ricchezza del pensiero di uno scienziato della storia", L'Osservatore Romano, 27 luglio 1996, p. 3 [titolo redazionale (titolo originale: “'Il diario segreto' di Gaetano De Sanctis")].

33. "Uno storico della latinità al servizio dell'umanesimo", L'Osservatore Romano, 4 dicembre 1996, p. 3 [titolo redazionale (titolo originale: "Ricordo di Pierre Grimal")].

34. "Presentazione", in L. Polverini (a cura di), Erudizione e antiquaria a Perugia nell'Ottocento, [Atti del Convegno (Acquasparta, 28-30 maggio 1990)], Incontri perugini di storia della storiografia antica e sul mondo antico, V, Napoli, 1998, p. 7-12.

35. "Vermiglioli, Fabretti, Conestabile fra biografia e storia", ibid., p. 127-144.

36. "Una lettera di Borghesi a Niebuhr (e l'iscrizione CIL X 7845)", in P. KNeIssL, V. Losemann (hrsg. von), Imperium Romanum. Studien zu Geschichte und Rezeption. Festschrift für Karl Christ zum 75. Geburtstag, Stuttgart, 1998, p. 571-581.

37. "La Deputazione umbra e la storia locale italiana. Gli studi di storia antica", in P. Pimpinelli, M. Roncetti (a cura di), Una regione e la sua storia, Atti del Convegno celebrativo del Centenario della Deputazione, 1896-1996 (Perugia, 19-20 ottobre 1996), Perugia, 1998, p. 63-78.

38. "[Prefazione]", in M. C. Spadoni, Reate, II, L'antiquaria, Pisa-Roma, 1998, p. 3.

39. "Storia romana e storia contemporanea: 'Il più antico trionfo romano sui Germani'", in L. Canfora (a cura di), Studi sulla tradizione classica per Mariella Cagnetta, RomaBari, 1999 , p. 437-450.

40. "Rostovzev e De Sanctis", in A. Marcone (a cura di), Rostovtzeffe l'Italia, [Atti del Convegno (Gubbio, 25-27 maggio 1995)], Incontri perugini di storia della storiografia antica e sul mondo antico, IX, Napoli, 1999, p. 97-113.

41. "Pavan e la Dalmazia", in L. BraccesI, S. Graciotti (a cura di), La Dalmazia e l'altra sponda. Problemi di archaiologhía adriatica, [Atti del Convegno (Venezia, 16-17 gennaio 1996)], Firenze, 1999, p. 3-12.

42. "Albino Garzetti e la storia antica", in L. MeLi Bassi (a cura di), Mons Braulius. Studi storici in memoria di Albino Garzetti, Sondrio, 2000, p. 17-25. 
43. "Ruggero Fauro Rossi e la storia romana della Tarda Repubblica", in G. BANDELLI (a cura di), La Scuola triestina di Storia antica, Atti dell'Incontro di studio in onore di Filippo Càssola e Ruggero Fauro Rossi (Trieste, 16 novembre 1998), Quaderni Giuliani di Storia 21 (2000), 2, p. 247-256.

44. "L'impero romano - antico e moderno", in B. NÄF (hrsg. von), Antike und Altertumswissenschaft in der Zeit von Faschismus und Nationalsozialismus, Kolloquium Universität Zürich (14.-17. Oktober 1998), Mandelbachtal-Cambridge, 2001, p. $145-163$.

45. "Introduzione", in L. Polverini (a cura di), Aspetti della storiografia di Ettore Pais, [Atti del Convegno (Acquasparta, 25-27 maggio 1992)], Incontri perugini di storia della storiografia antica e sul mondo antico, VII, Napoli, 2002, p. 7-19.

46. "Imitatio Caesaris. Cesare e Alessandro, Napoleone e Cesare", in A. Barzano, C. Bearzot, F. Landucci, L. Prandi, G. Zecchini (a cura di), Modelli eroici dall'antichità alla cultura europea, Roma, 2003, p. 403-414.

47. "Le ragioni di un convegno (e di questo volume)", in L. Polverini (a cura di), Arnaldo Momigliano nella storiografia del Novecento, [Atti del Convegno (Spoleto, 31 maggio - 2 giugno 1999)], Incontri perugini di storia della storiografia antica e sul mondo antico, XI, Roma, 2006, p. 1-8.

48. "Momigliano e De Sanctis", ibid., p. 11-35.

49. "Amicizia e storiografia nel carteggio Beloch-Meyer", in C. Bonnet, V. KRINGS (éds.), S'écrire et écrire sur l'Antiquité. L'apport des correspondances à l'histoire des travaux scientifiques, Grenoble, 2008, p. 105-119.

50. "Arnaldo Momigliano", in V. Losemann (hrsg. von), Alte Geschichte zwischen Wissenschaft und Politik. Gedenkschrift Karl Christ, Wiesbaden, 2009, p. 163-179.

51. "Per la storia della 'Geografia antica' in Italia (e in Germania)", Geographia antiqua 18 (2009), p. 195-197.

52. "La storia economica nell'insegnamento di Giulio Beloch", Rivista Storica Italiana 121 (2009), 3, p. 1232-1245.

53. "Una lettera di Beloch a Mommsen (e l'iscrizione CIL X 3702)", Athenaeum 98 (2010), 1, p. 267-270.

54. "Karl Christ", Archivio di storia della cultura 23 (2010), 13-17.

55. "Karl Christ", Gnomon 82 (2010), 5, p. 476-479.

Voci del Grande Dizionario Enciclopedico UTET, I-XX

Quarta edizione, Torino, 1984-1991 (Ristampe successive)

56. Beloch, Karl Julius, III (1985), p. 163-164.

57. Bury, John Bagnell, ibid., p. 840.

58. Curtius, Ernst, VI (1986), p. 155.

59. Durry, Marcel, VII (1987), p. 19.

60. Fraccaro, Plinio, VIII (1987), p. 724. 
61. Frank, Tenney, ibid., p. 818.

62. Gelzer, Matthias, IX (1987), p. 194.

63. Kornemann, Ernst, XI (1988), p. 671-672.

64. Marrou, Henri-Irénée, XIII (1989), p. 74-75.

65. Meyer, Eduard, ibid., p. 547-548.

66. Piganiol, André, XV (1989), p. 958.

67. Pöhlmann, Robert von, XVI (1990), p. 194.

68. Rostovcev, Michail Ivanovič, XVII (1990), p. 823-824.

69. Schwegler, Friedrich Karl Albert, XVIII (1990), p. 349.

70. Seeck, Otto, ibid., p. 478.

71. Syme, sir Ronald, XIX (1991), p. 651.

72. Veith, Georg, XX (1991), p. 793.

Edizioni di volumi

73. G. De Sanctis, Scritti minori, Novamente editi da A. Ferrabino e S. Accame, VI, Recensioni - Cronache e commenti, 1-2, [a cura di L. Polverini], Roma, 1972.

74. G. De Sanctis, La guerra sociale, Opera inedita, a cura di L. Polverini, Firenze, 1976.

75. Aspetti della storiografia di Giulio Beloch, [Atti del Convegno (Acquasparta, 19-21 maggio 1986)], Incontri perugini di storia della storiografia antica e sul mondo antico, I, Napoli, 1990.

76. Lo studio storico del mondo antico nella cultura italiana dell'Ottocento, [Atti del Convegno (Acquasparta, 30 maggio $-1^{\circ}$ giugno 1988)], Incontri perugini di storia della storiografia antica e sul mondo antico, III, Napoli, 1993.

77. Erudizione e antiquaria a Perugia nell'Ottocento, [Atti del Convegno (Acquasparta, 28-30 maggio 1990)], Incontri perugini di storia della storiografia antica e sul mondo antico, V, Napoli, 1998.

78. Aspetti della storiografia di Ettore Pais, [Atti del Convegno (Acquasparta, 25-27 maggio 1992)], Incontri perugini di storia della storiografia antica e sul mondo antico, VII, Napoli, 2002.

79. Arnaldo Momigliano nella storiografia del Novecento, [Atti del Convegno (Spoleto, 31 maggio - 2 giugno 1999)], Incontri perugini di storia della storiografia antica e sul mondo antico, XI, Roma, 2006. 